\title{
The dynamics of a dielectric liquid jet exposed to combined electrostatic and aerodynamic forces
}

\author{
Tushar Ahmed ${ }^{1}$, Agisilaos Kourmatzis*1, Assaad R. Masri ${ }^{1}$ \\ ${ }^{1}$ School of Aerospace, Mechanical and Mechatronic Engineering, \\ The University of Sydney, NSW 2006, Australia. \\ *Corresponding author email : agisilaos.kourmatzis@sydney.edu.au
}

\begin{abstract}
Electrostatic atomization can complement air-blast methods by enabling control of the dispersion of the spray as well as the motion and size of the droplets. In this contribution, a hybrid air-blast electrostatic atomizer is utilized to study the dynamics of a liquid jet exposed to a combination of air assistance and electrostatic forces. High speed microscopic imaging is used to track the interface of a liquid jet as a function of time. A range of Weber numbers (from 2.4-11.2) and different charge per unit volumes, are tested. The location of the liquid core is traced using an in-house particle tracking velocimetry (PTV) technique. The results indicate a much more erratic fluctuation in liquid jet motion upon inclusion of electric charge and this remains true over the range of Weber numbers tested. Examining the mean and standard deviation of liquid jet position indicates that charge has a noticeable influence on the transient dynamics of the jet over the range of We tested.
\end{abstract}

\section{Keywords}

Electrostatic atomization, air-blast atomization, PTV.

\section{Introduction}

Charge injection atomization is a liquid fragmentation process that has been studied extensively over the last few decades [1-6]. Briefly, this technique works through the introduction of a unipolar charge into an electrically insulating dielectric liquid [6]. Injection of charge is achieved through two electrodes separate by a distance $(\mathrm{L})$. One electrode is held at a high negative voltage, whilst the other is grounded. The grounded electrode also contains the orifice through which the liquid flows into the primary atomization zone. Upon exiting the orifice, the liquid charge can atomize due to the repulsive Coulombic force overcoming the surface tension force of the liquid [6]. Recent work in this field has focused on understanding the importance of the electrode geometry [7], liquid injection conditions, and liquid properties for the simple case of an injector with no aerodynamic assistance [8-11]. Information related to droplet size and velocity is now well documented, and it is understood that the droplet size correlates with spray specific charge [12]. Previous work by Yarin and co-workers [13] has also shed light on the formation of instabilities in electrified liquid jets.

In many practical applications, such as in turbulent spray combustion, it is necessary to introduce a high volumetric flow-rate of air to achieve a burnable mixture [14]. If a charged liquid jet is exposed to a high speed gas stream, it will not only atomize due to Coulombic repulsion, but also due to aerodynamic assistance. There has been minimal work done in studying the interaction between aerodynamic shear and electrostatic atomization of dielectric liquids, however recent work by Shrimpton and co-workers [15] has demonstrated that the effect of charge diminishes with aerodynamic Weber number (at a value of approximately We 20) and this was confirmed through follow up work by these authors [16]. Work by Ahmed et al. [16] has demonstrated that charge generates a greater population of droplets over a range of aerodynamic Weber numbers and notable influences on the wavelength of 
instabilities on the primary liquid jet were also noted [16]. In particular, a broadening in the probability density function of wavelength was highlighted with increasing charge [16]. Despite recent efforts in this area, it is still unclear how the addition of charge on the surface of a liquid jet can influence transient atomization phenomena, and this paper makes an initial contribution in this space. In particular, we focus on analysing the "flapping" of an electrified dielectric liquid jet exposed to a co-flowing air over a range of Weber numbers. Through analysis of jet position, the contribution aims to provide some initial insights into spray specific charge, and Weber number influence the dynamics of a liquid jet close to the injecting nozzle.

\section{Methodology}

Figure 1 represents the cross-sectional view of the hybrid air-blast electrostatic atomizer that has been used in this study. The charging of the liquid is done by supplying a high voltage through a long electrode and allowing the liquid to pass through the inter-electrode gap as shown in the detailed view of Figure 1 . The supply voltage is varied from $-5 \mathrm{kV}$ to $-9 \mathrm{kV}$ with an increment of $-1 \mathrm{kV}$. The atomizer is grounded via the air-blast tube, which is connected to the brass nozzle tip (part\#3) through the metal centering sleeve (part\#2). The fuel is supplied from a pressurized tank (see Fig. 2). The orifice diameter used is $310 \mu \mathrm{m}$ and the liquid jet velocity is maintained as $10 \mathrm{~m} / \mathrm{s}$. The fuel flow rate is controlled by a rotameter and an inline fuel filter is used to remove unwanted particles from the fuel.

A backlit microscopic imaging technique is implemented in this analysis and two high-speed Edgewave pulsed diode lasers are used as the light source. Images are taken using a highspeed LaVision Star 6 camera at a rate of $5 \mathrm{kHz}$. Two frames are captured at an interval of 20 $\mu$ s and altogether 2000 image sets are stored for each case. As per previous work by these authors, it is confirmed that these 2000 images are taken over a long enough interval of time to enable investigation of multiple break-up time scales [17]. Further operational details of the atomizer can be found in [16]. The Weber number is calculated based on the following equation and in this contribution, four different Weber numbers namely $2.4,5.2,8.2$, and 11.2 are tested.

$$
W e_{\text {gas }}=\frac{\rho_{\text {gas }}\left(U_{\text {gas }}-U_{\text {liquid }}\right)^{2} D_{\text {liquid }}}{\sigma}
$$

The image processing method is detailed extensively in previous contributions by these authors $[16,18]$. The reader should note that the mean velocity $\left(U_{\text {gas }}\right)$ is calculated based on the annular area between the liquid jet and the inner airblast tube. It is highly likely that the local velocity adjacent to the liquid jet is higher than this mean estimate, but given the lack of further measurements, we use this as an approximation. The applied voltages and corresponding spray specific charge information are listed in Table 1.

Table 1 - Operating parameters in EHD mode.

\begin{tabular}{c|c}
\hline $\begin{array}{c}\text { Applied } \\
\text { Voltage, kV }\end{array}$ & $\begin{array}{c}\text { Spray Specific } \\
\text { Charge, } \mathrm{C} / \mathrm{m}^{3}\end{array}$ \\
\hline-5 & 0.042 \\
-6 & 0.083 \\
-7 & 0.14 \\
-8 & 0.23 \\
-9 & 0.33
\end{tabular}




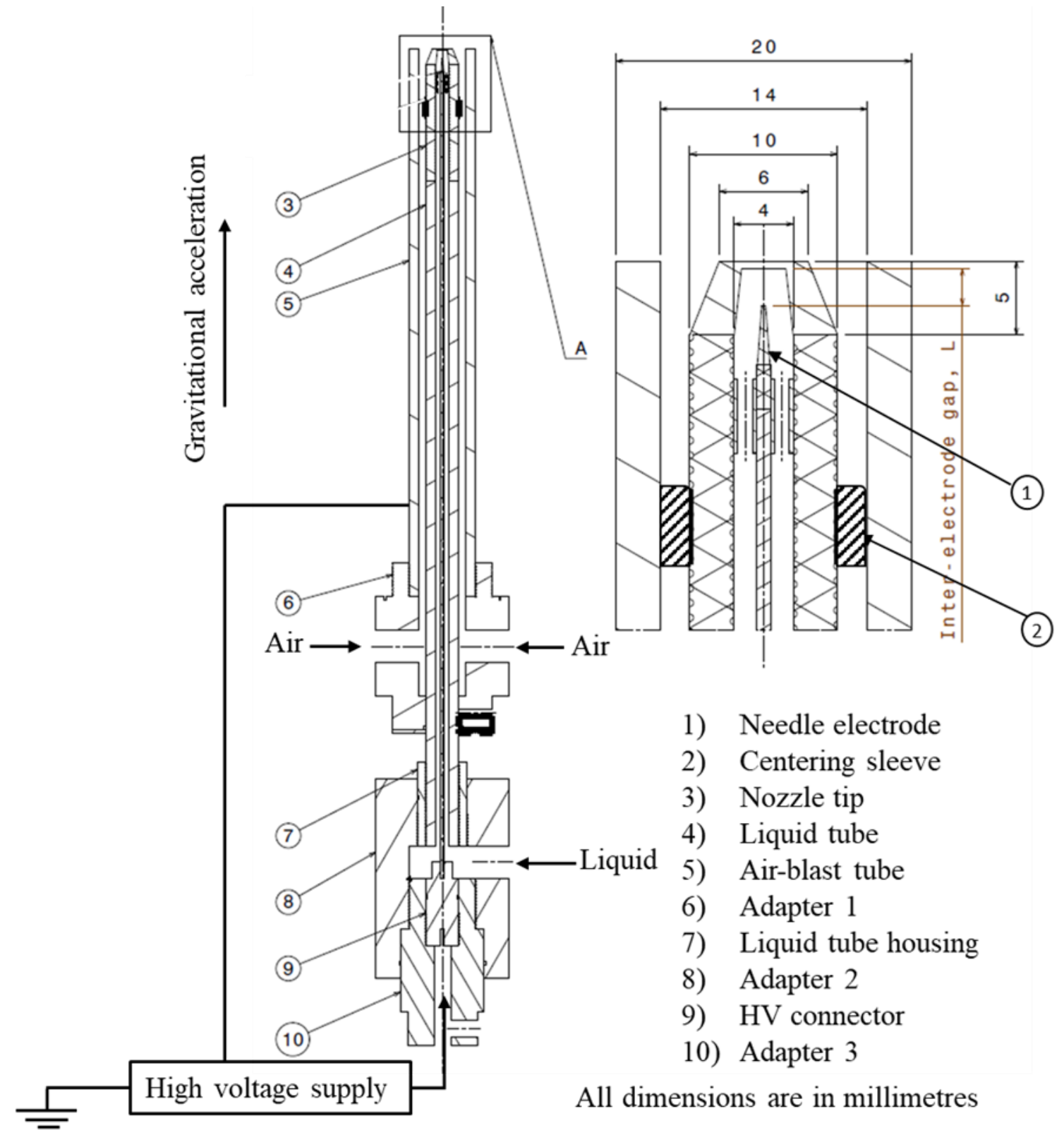

Figure 1. Cross-section of the hybrid atomizer [16].

\section{Results and Discussion}

Figure 3 illustrates a sample of an instantaneous microscopic high-speed image just downstream of the liquid orifice, where no atomization is present. The distance between the liquid jet and the center of the image is identified as this metric is used to characterize the transient degree of "flapping" of the liquid jet with respect to a fixed position. The reader should take note that the distance statistics (mean and standard deviation) provided in Figs. 4 onwards are calculated from the absolute value of distance. Therefore, a positive mean for example, does not indicate that the liquid jet has a predominant asymmetry. 


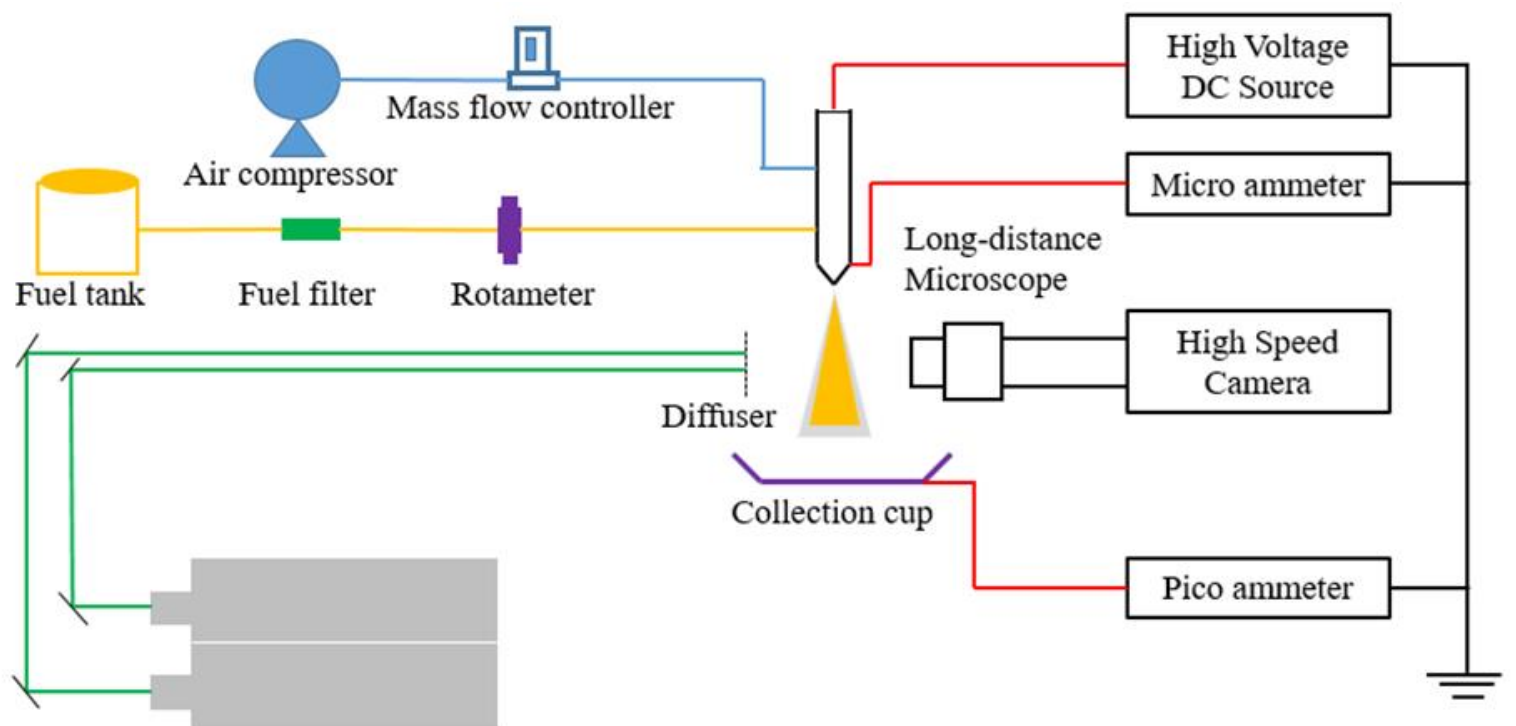

Edgewave high speed diode lasers

Figure 2. Schematic of the experimental arrangement [16].

Figure 4 shows the location of the liquid jet (determined as shown in Figure 3) at a We=2.4 with and without charge, with respect to time. It is visible that the application of charge results in a higher mean value of distance from the centre. This clearly indicates that there is a greater propensity for the liquid jet to flap, however it is also noted that the process is much more erratic than in the uncharged case. This enables an isolation of the influence of charge on flapping for a fixed Weber number. Fluctuations in the liquid jet with charge are noted to be no greater than 200um ( 0.65D $\left.D_{\text {liquid }}\right)$, and the degree of fluctuation changes with Weber number and charge which is examined next.

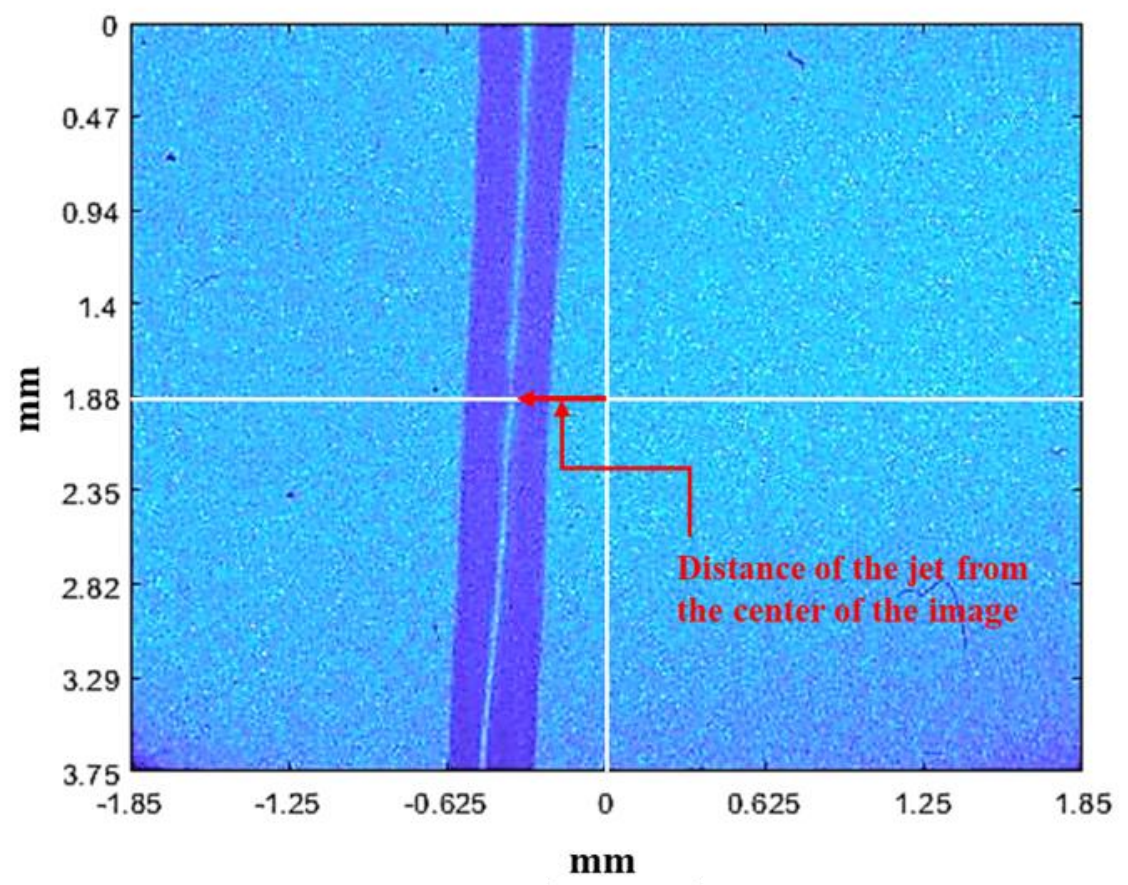

Figure 3. Microscopic high-speed image demonstrating the distance calculation. 


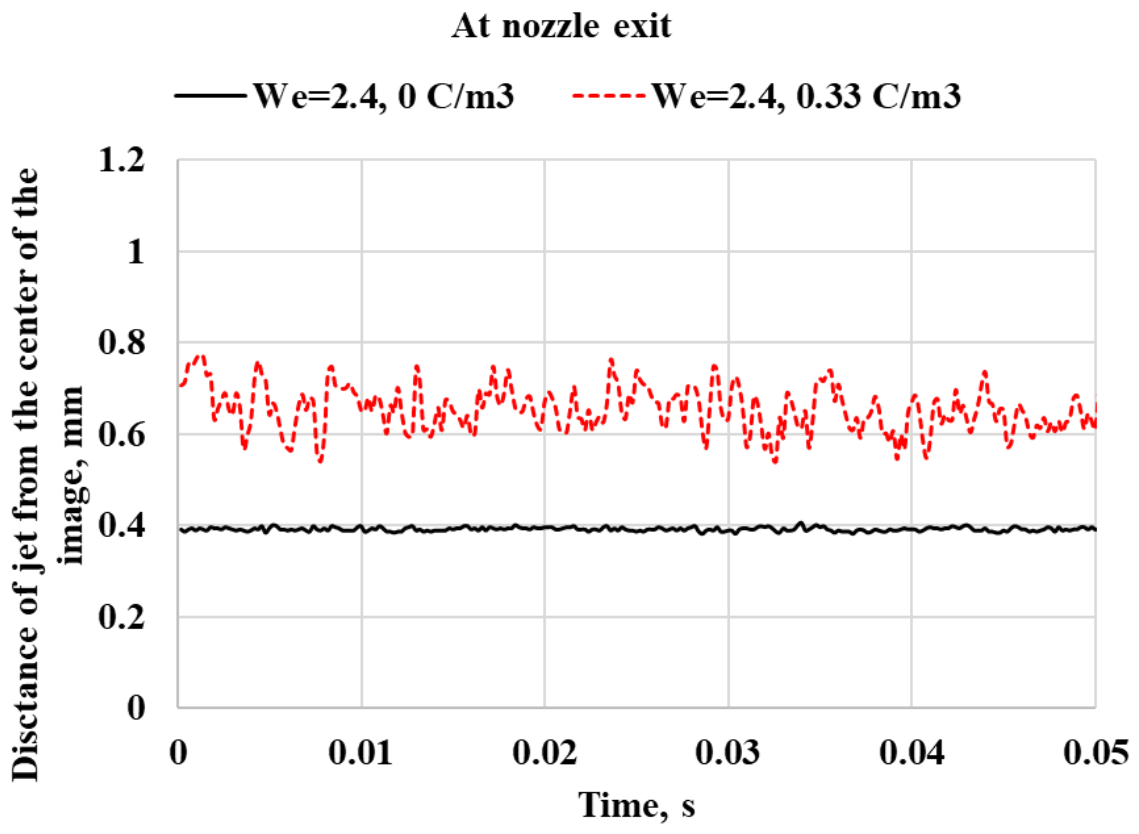

Figure 4. Liquid jet distance from the centre versus time.

Figure 5a shows the mean radial distance of the liquid jet from the center of the image versus axial location for different charge levels at $\mathrm{We}=2.4$. The charge is affecting the position of the jet at all axial locations. However, the effect of the charge in moving the liquid jet further outward compared with the uncharged case is found to be most noticeable at and above 0.14 $\mathrm{C} / \mathrm{m}^{3}$ (corresponds to $-7 \mathrm{kV}$, see Table 1), with the maximum effect observed at $0.33 \mathrm{C} / \mathrm{m}^{3}$ which is the highest level of charge applied in this study. At $-5 \mathrm{kV}$ and $-6 \mathrm{kV}$, minimum mean distances are recorded throughout the studied range of axial locations, indicating that the jet moves inward at those voltages rather than going outward, however the precise reasons for this must be subject to further study. It is noteworthy that for the highest value of charge, the mean distance decreases past an axial location of $6 \mathrm{~mm}$. Further work is also required to understand this phenomenon, however this location is just upstream of break-up and coincides with the region of highest standard deviation, as shown in Fig. 5b and Fig. 6.

The standard deviation of the data sets for each charge level has been plotted against axial locations in Figure 5b. The findings interestingly show that despite the unclear trend in the mean flapping distance with axial distance, it is very clear that charge has a proportionally stronger influence on the erratic nature of the flapping mode as the jet moves downstream. In an electrostatic atomizer, the injected charge transforms into surface charge, which can be redistributed through ionic mobility and charge convection. There is also an electric field that exists radial to the liquid jet[19]. The presence of charge will accelerate the break-up process which will cause portions of the liquid jet be more affected by aerodynamic forces closer to the orifice. As a result, at a given downstream position, cases with more charge have undergone more break-up compared to the uncharged case, resulting in more instability and eventually, more rapid fragmentation[6, 16, 19].

To clarify further, the mean "spread" of the jet is not as consistently affected as the randomness of the jet. This must be somehow related to the combined influence of aerodynamic forces and Coulombic repulsion, which would be more obvious as the liquid jet 
migrates closer to the break-up location [16]. This statement is consistent with previous work by these authors showing that the influence of aerodynamic Weber number on instabilities is more prominent close to the break-up point, rather than closer to the nozzle [20], where in the former region, the liquid jet Reynolds number dominates instability formation.

$\mathrm{We}=\mathbf{2 . 4}$
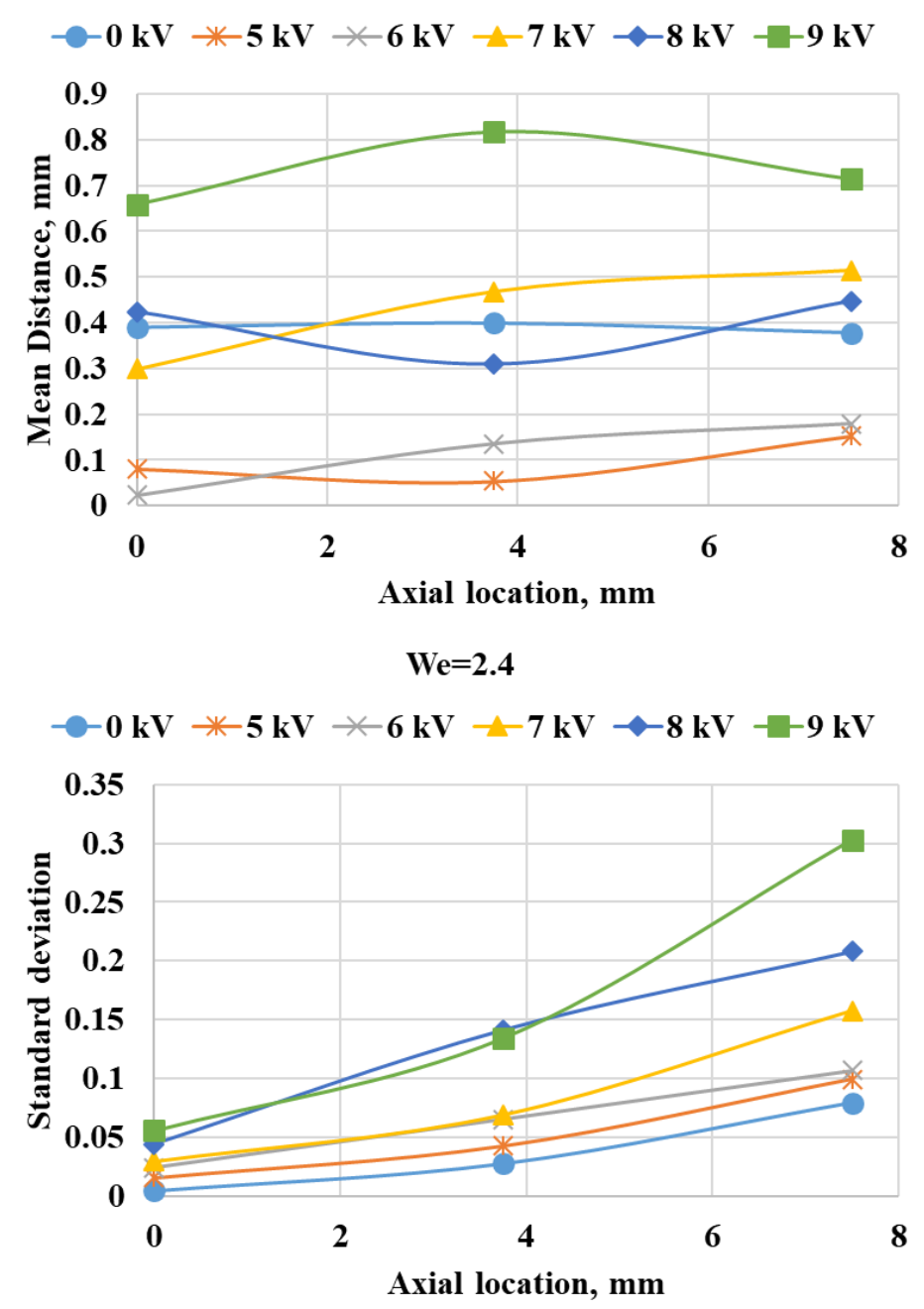

Figure 5. (a) Mean radial distance of the liquid jet at various charge levels in different axial locations, (b) Standard deviation of those data sets at that locations.

Figure 6 presents the effect of charge on the mean distance (standard deviation shown as error bars) for various Weber numbers. As observed in [16], at most of the axial locations, the maximum effect of charge in moving the jet further outward is obtained for the lowest Weber number case, namely $\mathrm{We}=2.4$, and the effect of charge reduces gradually with increasing Weber number. The standard deviation data indicates that for the same charge level at $\mathrm{We}=2.4$, the liquid jet is flapping more erratically compared to other Weber numbers that have been tested. Unlike with Fig. 5 however, the effect of charge with mean distance shows a clear trend for the highest Weber number case, and again this is consistent with the notion that aerodynamic forces dominate closer to the break-up zone. In this case, this indicates an interaction between the charge and aerodynamic forces that is noted far from the nozzle. 


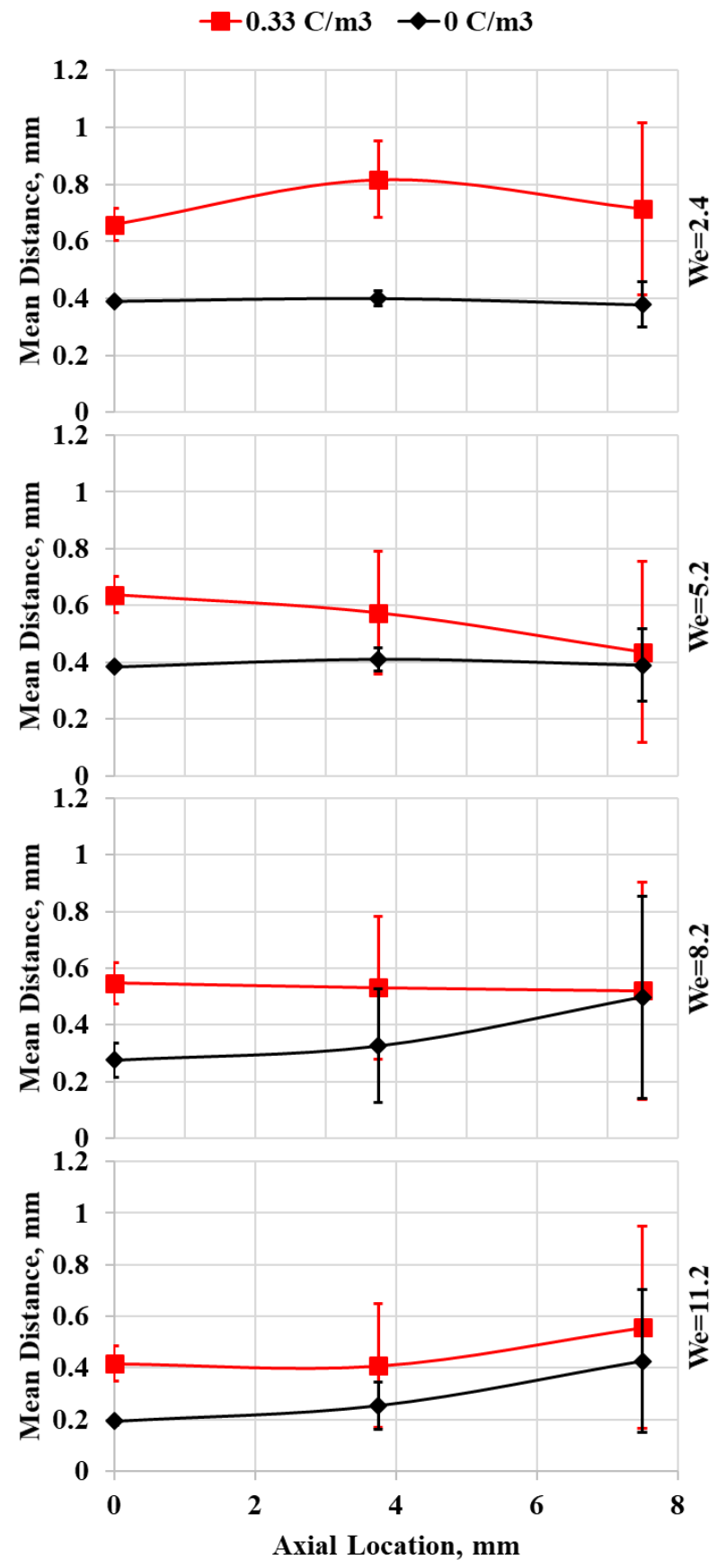

Figure 6. Mean radial distance of the liquid jet at various Weber numbers and axial locations: with and without EHD.

\section{Conclusions}

High speed microscopic imaging is used to quantify the dynamics of a liquid jet produced by a hybrid air-blast and electrostatic atomizer. The following key findings are obtained:

- Application of charge helps to make the liquid jet more unstable than the pure air-blast mode and the unsteadiness increases with increasing charge level.

- The lower Weber number case is generally found to be more affected by the charge in terms of the mean flapping distance.

- There is a clearer trend in the standard deviation of distance (related to the "erratic" nature of the flapping) with axial location when compared to the mean (related to the overall radial spread of the jet) vs. axial distance. 
Future work will focus on more detailed analysis of the transient behaviour of the liquid jet including computation of local dimensionless numbers, as well as quantifying the unsteady atomization process in terms of variation in droplet number vs. time as opposed to just liquid jet dynamics.

\section{Acknowledgments}

The work is funded by the Australian Research Council.

\section{References}

1. Kelly, A., The electrostatic atomization of hydrocarbons. J. INST. ENERGY, 1984. 57(431): p. 312-320.

2. Robinson, K.S., R.J. Turnbull, and K. Kim, Electrostatic spraying of liquid insulators. IEEE Transactions on Industry Applications, 1980(2): p. 308-317.

3. Kim, K. and R. Turnbull, Generation of charged drops of insulating liquids by electrostatic spraying. Journal of Applied Physics, 1976. 47(5): p. 1964-1969.

4. Jido, M., Study of electrostatic charged droplets: Part 1. J. Jpn. Soc. Chem. Eng, 1986. 40: p. 24-33.

5. Shrimpton, J.S., Atomization, combustion, and control of charged hydrocarbon sprays. ATOMIZATION SPRAY 2001. 11(4): p. 365-396.

6. Shrimpton, J., Charge injection systems: physical principles, experimental and theoretical work. 2009, Berlin Heidelberg: Springer

7. Kourmatzis, A., et al., Electrical and spray characteristics of a multiorifice charge-injection atomizer for electrically insulating liquids. 2010. 20(4).

8. Rigit, A. and J. Shrimpton, Electrical performance of charge injection electrostatic atomizers. ATOMIZATION SPRAY 2006. 16(4).

9. Rigit, A. and J.S. Shrimpton, Spray characteristics of charge injection electrostatic atomizers with small-orifice diameters. ATOMIZATION SPRAY 2006. 16(4).

10. Al-Ahmad, G., et al., Electrical performance of a charge-injection atomizer using viscous organic oils. Atomization Sprays, 2009. 19(6).

11. Singh, G., et al., Effect of electric charge and temperature on the near-field atomization of diesel and biodiesel. Fuel, 2019. 241: p. 941-953.

12. Shrimpton, J.S. and A. Yule, Drop size and velocity measurements in an electrostatically produced hydrocarbon spray. J FLUID ENG-T ASME, 1998(120(3)): p. 580-585.

13. Yarin, A.L., S. Koombhongse, and D.H. Reneker, Bending instability in electrospinning of nanofibers. J Journal of applied physics, 2001. 89(5): p. 3018-3026.

14. Masri, A., Challenges for turbulent combustion. Proceedings of the Combustion Institute, 2021. 38(1): p. 121-155.

15. Shrimpton, J., Mashayek F, Haroutunian N, Schick RJ, Air-assisted electrohydrodynamic atomization of dielectric liquids. ICLASS 2018.

16. Ahmed, T., A. Kourmatzis, and A.R. Masri, Atomization behaviour of a hybrid air-blastelectrostatic atomizer for spray combustion. FUEL, 2021. 288: p. 119716.

17. Kourmatzis, A. and J. Shrimpton, Electrohydrodynamic inter-electrode flow and liquid jet characteristics in charge injection atomizers. EXP FLUIDS, 2014. 55(3): p. 1-13.

18. Kourmatzis, A., P.X. Pham, and A.R. Masri, A two-angle far-field microscope imaging technique for spray flows. Measurement Science Technology, 2017. 28(3): p. 035302.

19. Turnbull, R.J., On the instability of an electrostatically sprayed liquid jet. IEEE transactions on industry applications, 1992. 28(6): p. 1432-1438.

20. Singh, G., et al., Instability growth and fragment formation in air assisted atomization. Journal of Fluid Mechanics, 2020. 892. 